09

\title{
Географическая привязка кадров широкоугольных оптических систем
}

\author{
(C) T.Е. Сыренова, ${ }^{1}$ А.Б. Белецкий, ${ }^{1}$ Р.В. Васильев ${ }^{1,2}$ \\ ${ }^{1}$ Институт солнечно-земной физики СО РАН, \\ 664033 Иркутск, Россия \\ ${ }^{2}$ Иркутский государственный университет, \\ 664003 Иркутск, Россия \\ e-mail: angata@mail.iszf.irk.ru
}

Поступило в Редакцию 7 июня 2021 г.

В окончательной редакции 20 июля 2021 г.

Принято к публикации 10 августа 2021 г.

Описана методика привязки кадров широкоугольных оптических систем, предназначенных для регистрации собственного излучения атмосферы Земли, к географическим координатам. Методика основана на автоматической процедуре выделения и идентификации звезд на кадрах и последующей географической привязке. Приведен пример использования методики для расчета характеристик долгоживущего метеорного следа по данным наблюдений двух пространственно-разнесенных широкоугольных оптических систем.

Ключевые слова: географическая привязка, широкоугольная камера, идентификация звезд, атмосферные эмиссии, долгоживущий метеорный след.

DOI: 10.21883/JTF.2021.12.51765.172-21

\section{Введение}

При наблюдениях за верхней атмосферой Земли с помощью оптических инструментов необходима географическая привязка для оценки высоты и других пространственных параметров наблюдаемых событий. Для этого нужно знать точное соответствие пикселей кадров оптических систем координатам Земли. Это можно осуществить с помощью привязки к объектам с известными координатами, например, звездам. В настоящее время для идентификации звезд, полученных с помощью оптических инструментов, используемых в астрономических наблюдениях, разработаны приложения, например, Izmced [1], Astrometry.net [2]. Но для широкоугольных оптических систем данные приложения не работают. Широкоугольные камеры, предназначенные для регистрации пространственного распределения интенсивности атмосферных эмиссий, являются достаточно удобным и доступным наземным средством регистрации различных событий, происходящих в верхней атмосфере Земли. Задача выделения и идентификации звезд на кадре усложняется тем, что вышеуказанные оптические системы регистрируют оптическое излучение в узком спектральном диапазоне. Так как в регистрируемых диапазонах высвечиваются достаточно мощные атмосферные эмиссии, то отношение интенсивности звезды в этом спектральном диапазоне к фону (основной вклад в который дает атмосферная эмиссия), существенно ниже такого же отношения в данных оптических систем с широким спектральным регистрируемым диапазоном.

При этом возможность использования двух и более разнесенных на некоторое расстояние инструментов позволяет определять пространственные параметры наблю- даемых событий [3-5]. Оптические наблюдения с использованием разнесенных в пространстве инструментов проводились еще в начале ХХв. для наиболее точного определения пространственных характеристик метеоров, полярных сияний или серебристых облаков [6-10]. В работе [10] описан оригинальный метод, базирующийся на анализе стереоизображений, для получения средней высоты и измерения основных параметров акустикогравитационных волн в мезосфере, таких как горизонтальная длина волны, временной период и вертикальная амплитуда форм волн. Кроме этого, при помощи оптических инструментов были выполнены исследования светящихся образований, возникающих в результате работы нагревных стендов. Регистрация искусственного свечения производилась в двух разнесенных пунктах камерами всего неба на длине волны 630 и $557.7 \mathrm{~nm}$. В результате были получены изображения светящихся областей, позволившие стереоскопическим методом определить пространственные параметры образований, их связь с параметрами мощной радиоволны, оценить количество дополнительной ионизации, вызванной работой нагревного стенда $[11,12]$.

\section{1. Описание методики}

При обработке изображений важным аспектом является точное соответствие координат пикселей и проекций изображений [13]. Вместе с тем на кадрах, полученных с помощью оптических систем с объективами типа „рыбий глаз“, имеют место значительные искажения изображений. В дополнение к искажениям вследствие оптических аберраций погрешность в определении координат может 

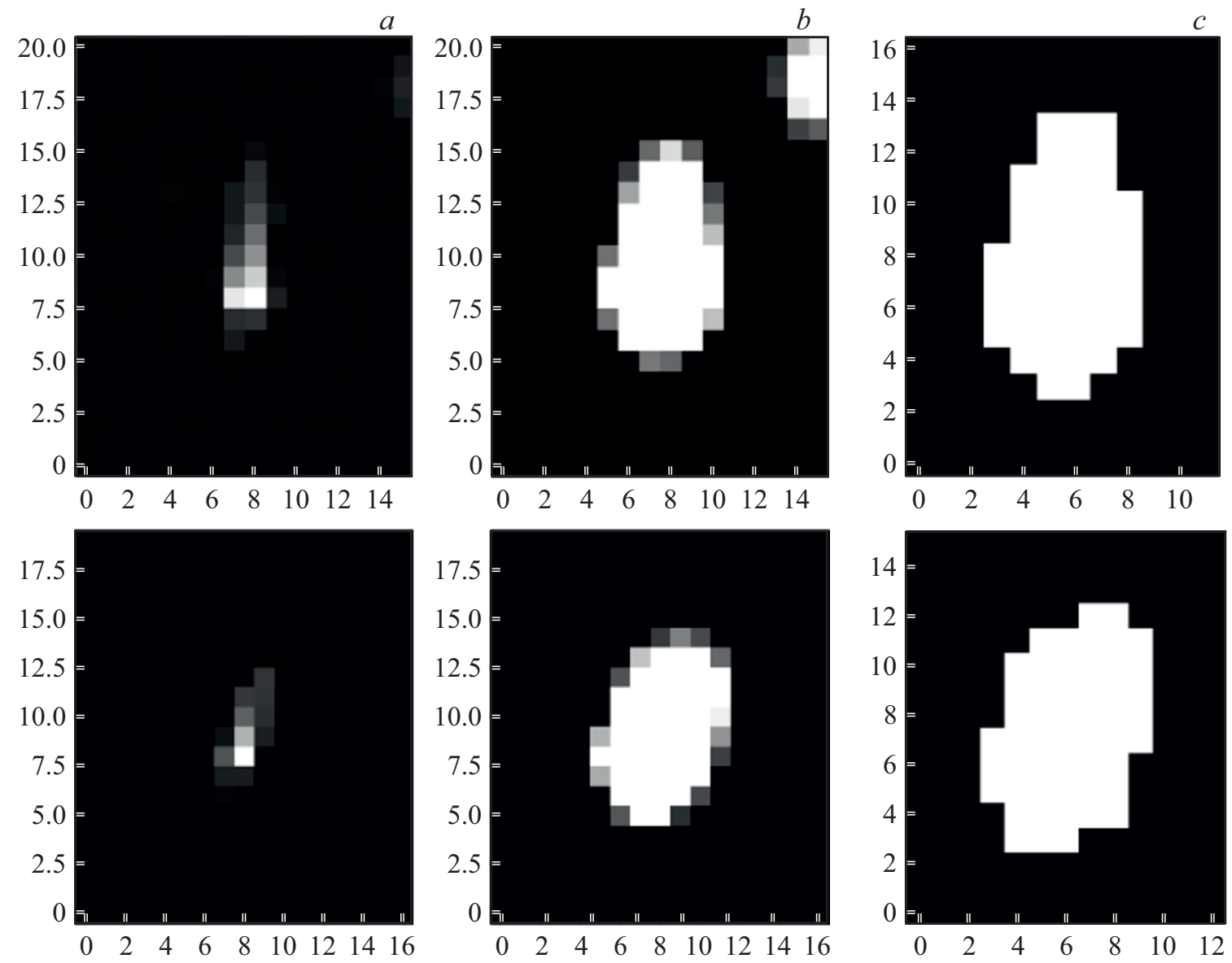

Рис. 1. Отдельные участки кадра оптической системы KEO Sentinel, полученного 3 декабря 2016 г. в 16:50 UT без обработки (a), контрастированные $(b)$ и группа пикселей, определенные алгоритмом, как изображение звезды $(c)$.

вносить и децентрализация - разница в положении истинного зенита и центра изображения. Этому вопросу посвящено достаточно большое количество работ, которые включают калибровку камер с использованием различных моделей с целью избавления от указанных искажений, например [14-18].

Предложенная в настоящей работе методика включает геометрическую калибровку камеры с подбором коэффициентов, учитывающих описанные выше аберрации, а также нахождение географических координат для каждого пикселя изображения широкоугольной камеры для любой высоты.

Географическая привязка выполнялась с использованием реперных точек, идентифицированных автоматическим алгоритмом как звезды. Первым этапом алгоритма выделения звезд на кадрах является удаление шумовых пикселей. Для этого изображение выравнивается с помощью удаления низкочастотного тренда - из исходного кадра отнимается кадр, сглаженный фильтром Гаусса [19] по двадцати пикселям. Затем выбираются одиночные пиксели со значениями интенсивности, превышающими некоторую пороговую величину, рассчитанную следующим образом:

$$
I_{\text {noise }}=I_{\text {mean }} K_{\text {noise }} \text {. }
$$

Коэффициент $K_{\text {noise }}$ подбирается для конкретной оптической системы эмпирически. Для KEO Sentinel $K_{\text {noise }}$ равен двум. Соседние пиксели, превышающие пороговое значение, объединяются в полигоны. Пиксель определяется как шумовой, если количество пикселей в полигоне не более одного. При определении пикселя как шумового ему присваивается среднее значение окружающих пикселей.

Далее проводится контрастирование изображения с помощью процедуры адаптивной гистограммной эквализации с ограничением контрастности. После этого проводится поиск полигонов связанных пикселей со значениями, превышающими пороговое $I_{\text {star }}$, и количеством пикселей в группе более $N_{\min }$ и менее $N_{\max }$. Значения $N_{\min }$ и $N_{\max }$ подбираются эмпирически для конкретной оптической системы. $N_{\min }$ позволяет ограничить звездную величину найденных групп пикселей. Например, для оптической системы KEO Sentinel значение $N_{\min }=5$ позволяет выбрать группы пикселей со звездными величинами ярче трех. Подбор значения $N_{\max }$ позволяет отсечь яркие объекты больших размеров, такие как Луна, подсвеченные Луной облака, блики на куполе и т. п. Для KEO Sentinel $N_{\max }=100$. На рис. 1 показаны участки кадра камеры с группами пикселей, 
определенные алгоритмом, как изображение звезды $(c)$, эти же участки на оригинальном кадре $(a)$ и после процедуры контрастирования $(b)$.

Для каждого объекта были сопоставлены азимут и высота в горизонтальной системе координат с центром в месте расположения камеры и соответствующие горизонтальный и вертикальный номер пикселя изображения.

Далее для сопоставления координат пикселя на изображении с соответствующим углом места и азимутом использовались выражения:

$$
\begin{gathered}
\phi(x, y)=\tan ^{-1}\left(\frac{y-y_{0}}{x-x_{0}}\right)+\phi_{0}, \\
\theta(x, y)=a r^{2}+b r+c, \\
r=\sqrt{\left(x-x_{0}\right)^{2}+\left(y+y_{0}\right)^{2}} .
\end{gathered}
$$

Выражение (1) определяет азимутальный угол с учетом произвольного поворота камеры на угол $\phi_{0}$, где $x, y$ - пиксельные координаты на изображении, $x_{0}$, $y_{0}$ - положение истинного зенита в этих координатах. Уравнение (2) определяет зенитное угловое расстояние пикселя, которое удобно записать при помощи расстояния между произвольным пикселем и истинным зенитом на матрице (3). Приведенная зависимость имеет квадратичный характер относительно расстояния (3) с постоянными коэффициентами $a, b$ и $c$. Подобные зависимости совместно с экспоненциальным представлением для широкоугольных камер использовались в работах [20,21]. Затем в последующих работах [22] было показано, что квадратичная зависимость является более точной и содержащей наименьшие ошибки. Связь между координатами (1)-(3) также можно представить в комплексном виде

$$
z=x+i y=\theta(x, y) \exp ^{i \phi(x, y)},
$$

где зависимости $\theta(x, y)$ и $\varphi(x, y)$ определяются выражениями (1)-(3). Используя реперные координаты звезд в горизонтальной системе и их соответствующие положения на матрице камеры, постоянные коэффициенты в (1)-(3) можно найти методом наименьших квадратов (МНК), используя (4) как модельную функцию. Минимизационная задача МНК с использованием (4) решалась при помощи алгоритма Левенберга-Марквардта [23]. В качестве функции ошибки использовался модуль разности (5) для угла места и азимута объекта из каталога РуEphem [24] и для горизонтальных координат, полученных из координат пикселя, соответствующего центру этого объекта на изображении камеры

$$
S=\sum_{n=0}^{M}\left|\theta_{n} e^{i \varphi_{n}}-\theta\left(x_{n}, y_{n}\right) e^{i \varphi\left(x_{n} y_{n}\right)}\right|
$$

для угла места и азимута $n$-го объекта из каталога звезд, и его соответствующих координат на изображении.
На рис. 2 показана часть кадра оптической системы KEO Sentinel 12 апреля 2018 г. Всего на полном кадре идентифицировано 29 звезд, совпавших со звездным каталогом, с яркостью от -1.44 до 1.98 .

На рис. 3 приведены траектории звезд, идентифицированных на кадре KEO Sentinel 3 декабря 2016 г.

Для кадров оптической системы KEO Sentinel для 3 декабря 2016 г. были получены следующие значения параметров выражений (2)-(4): $a=0.00000089$, $b=-0.29, \quad c=90.55, \quad \phi=264.30, \quad x_{0}=256.77$, $y_{0}=265.50$. На рис. 4 приведены ошибки определения углов места и азимутов звезд, идентифицированных на кадрах 3 декабря 2016 г. Для остальных параметров среднеквадратичные отклонения также достаточно малы, что говорит о том, что предлагаемый алгоритм достаточно устойчив.

Далее необходимо прийти к общей системе координат для двух камер. Наиболее удобно для дальнейшей работы камер и сопоставления полученных сведений с результатами наблюдений других инструментов (оптических и радиофизических) перейти к географическим координатам проекций изображения камеры на поверхность Земли. Позицию каждого пикселя изображения с использованием выражений (2)-(4) и полученных постоянных коэффициентов можно преобразовать в горизонтальные координаты. Для большинства геофизических задач наблюдения параметров верхней атмосферы нет необходимости использовать геоид в качестве модели Земли. Тогда решением прямой геодезической задачи на шаре [25] можно найти географические координаты проекции изображения с некоторой высоты на поверхность Земли. Исходными данными для этого являются географические широта и долгота точки наблюдений, азимут $\phi$, угол места $\theta$ и предполагаемая высота $h$, а сферическое расстояние $\sigma$ от местоположения камеры до спроецированной точки вычисляется по формуле

$$
\sigma=180-(90+\theta)-\arcsin \left(\frac{R_{\mathrm{E}} \sin (90+\theta)}{R_{\mathrm{E}}+h}\right),
$$

где $R_{\mathrm{E}}-$ радиус Земли.

Широта спроецированной точки определяется по формуле

$$
\varphi_{2}=\arcsin \left(\sin \varphi_{1} \cos \sigma+\cos \varphi_{1} \sin \sigma \cos \phi_{0}\right)
$$

где $\varphi_{1}$ - географическая широта точки наблюдений, $\phi_{0}$ - азимут.

Формула для нахождения долготы $\lambda_{2}$ спроецированной точки

$$
\lambda_{2}=\lambda_{1}+\operatorname{arctg}\left(\sin \sigma \frac{\sin \alpha_{1}}{\cos \varphi_{1}} \cos \sigma-\sin \varphi_{1} \sin \sigma \cos \alpha_{1}\right)
$$

где $\lambda_{1}$ - географическая долгота точки наблюдений. 


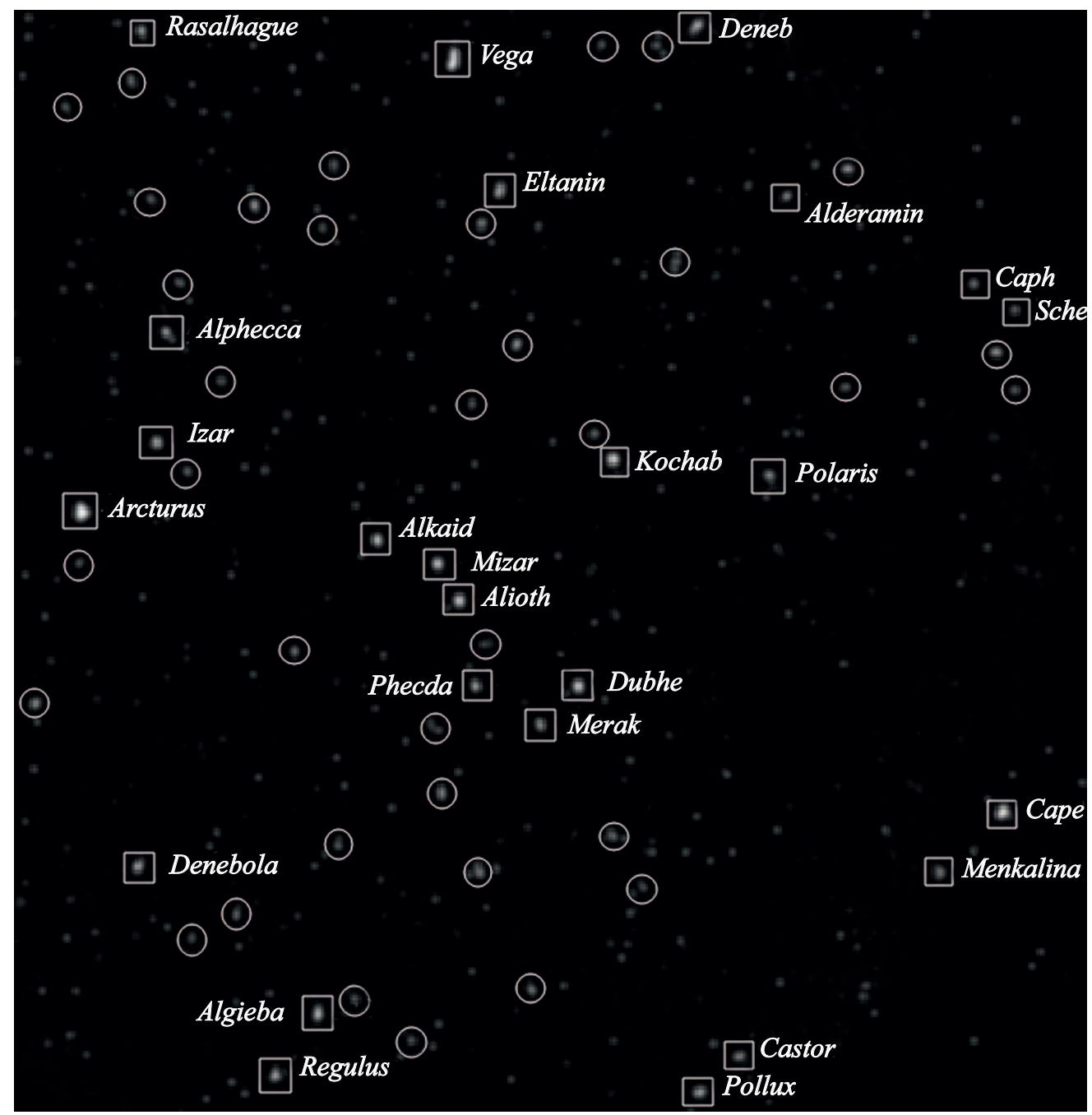

Рис. 2. Пример работы алгоритма идентификации звезд. Показана часть кадра оптической системы KEO Sentinel, зарегистрированного 12 апреля 2018 г. Кружками обозначены области кадра, идентифицированные как звезды, квадратами - совпавшие со звездным каталогом.

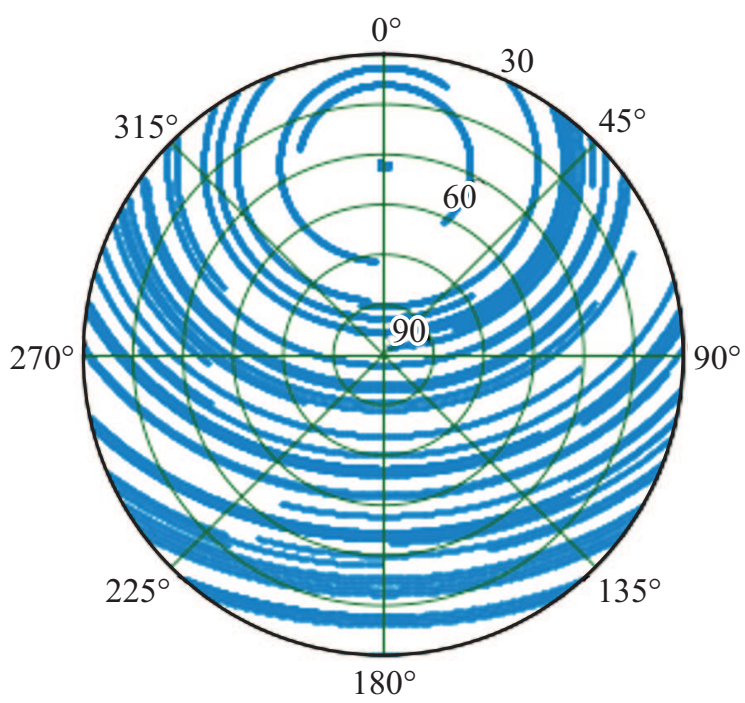

Рис. 3. Пример траекторий звезд, идентифицированных на кадре 3 декабря 2016 г.

\section{2. Результат применения предлагаемой методики}

Представленная в настоящей работе методика была апробирована на примере долгоживущего метеорного следа (ДМС), который наблюдался одновременно двумя широкоугольными камерами, пространственноразнесенными на расстояние около $150 \mathrm{~km}$. Первая камера - камера всего неба KEO Sentinel - расположена в Геофизической обсерватории (ГФО) ИСЗФ СО РАН, вблизи с. Торы $\left(51^{\circ} 48^{\prime}\right.$ с.ш., $103^{\circ} 04^{\prime}$ в. д., высота $\left.670 \mathrm{~m}\right)$ и предназначена для регистрации пространственной картины интенсивности эмиссии $630 \mathrm{~nm}$ (высота высвечивания 180-300 km). Направление визирования зенит, поле зрения $145^{\circ}$, время экспозиции 30-60 s. Полуширина пропускания интерференционного фильтра $\sim 2 \mathrm{~nm}$ [26]. Вторая широкоугольная цветная камера Allsky-340 производства SBIG расположена в Саянской 

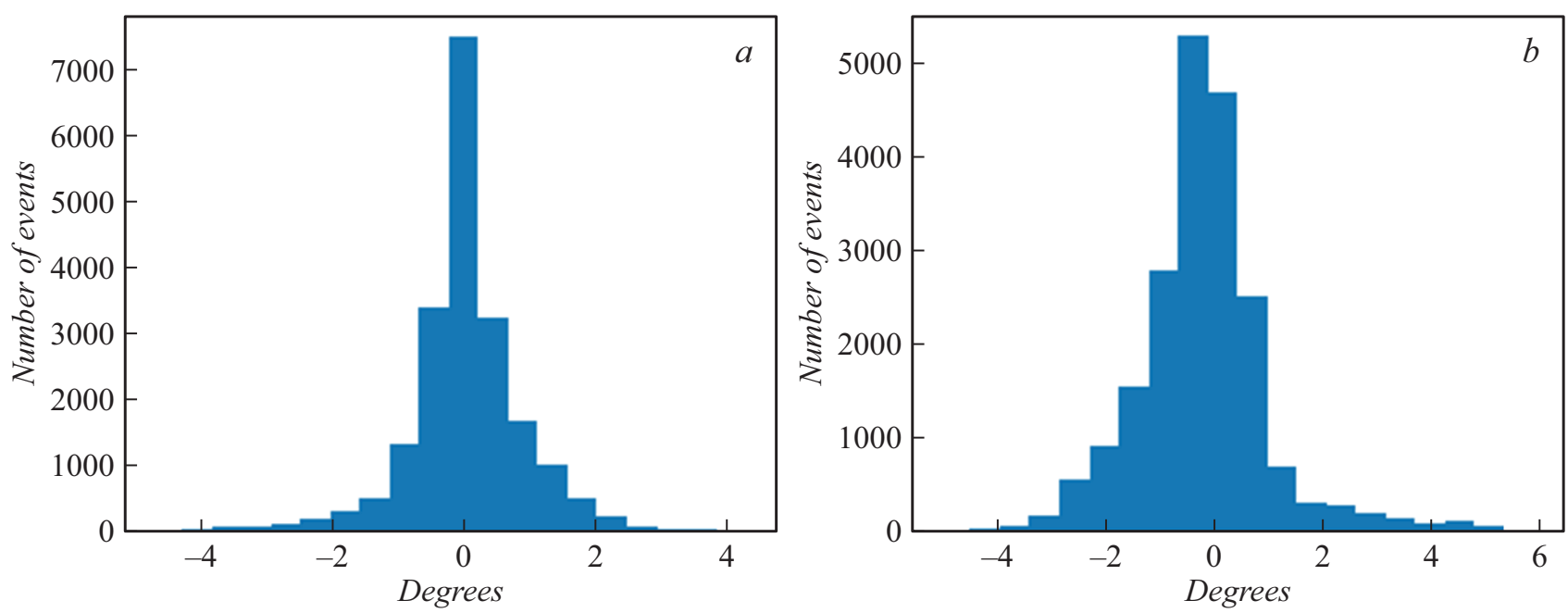

Рис. 4. Ошибки определения углов места $(a)$ и азимутов $(b)$ звезд, идентифицированных на изображениях, полученных 3 декабря 2016 г.

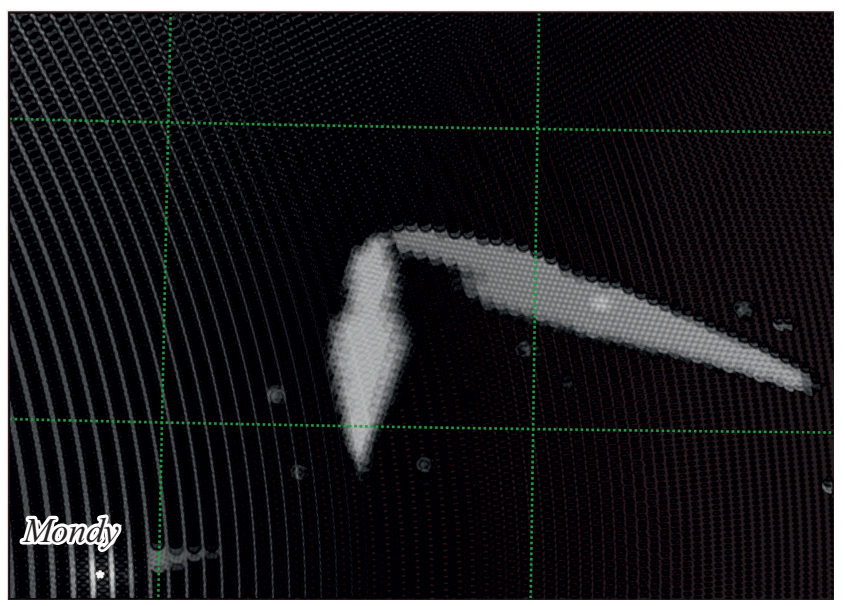

$101^{\circ} \mathrm{E}$ $101.5^{\circ} \mathrm{E}$

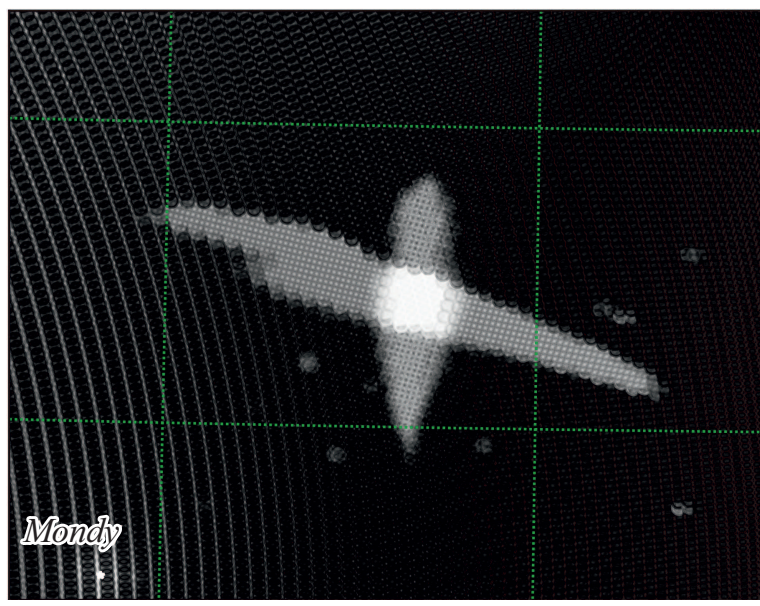

$101^{\circ} \mathrm{E}$

$101.5^{\circ} \mathrm{E}$

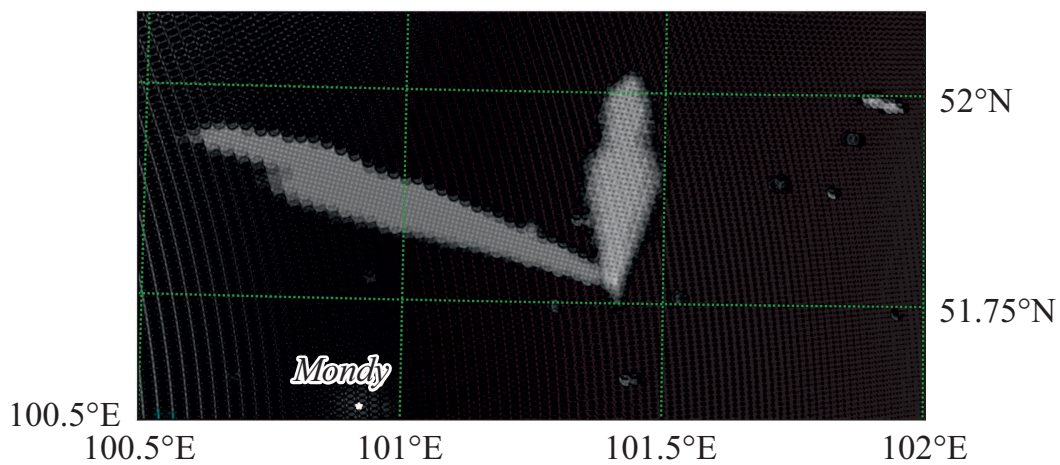

Рис. 5. Проекция метеора с камер KEO Sentinel и Allsky-340 на поверхность Земли с высоты 78, 92 и $111 \mathrm{~km}$ соответственно. На карте указан пункт наблюдения Mondy (Allsky-340), для которого наблюдаемое событие находится вблизи зенита. Наблюдательный пункт Tory (KEO Sentinel) находится за пределами карты.

солнечной обсерватории ИСЗФ СО РАН, вблизи с. Монды $\left(51^{\circ} 40^{\prime}\right.$ с. ш., $100^{\circ} 59^{\prime}$ в. д., высота $\left.1992 \mathrm{~m}\right)$.

Метеорный след продолжительностью 35-40 min был зарегистрирован после взрыва метеороида 18.11.2017 г. практически одновременно, в 22.23.19 UT.
Ранее уже проводилось исследование характеристик этого метеорного следа [27-29]. В работе [29] были определены пространственно-кинематические характеристики метеорного следа, выявлены особенности его эволюции. Значения высот загорания и погасания 
собственно метеора находились в диапазоне $75-120 \mathrm{~km}$, оценка блеска метеора дает значение абсолютной звездной величины около - $7.3 \mathrm{~m}$. Показано, что распространение всех участков долгоживущего метеорного следа происходит в одной плоскости на высоте около $90 \mathrm{~km}$ со скоростью около $320 \mathrm{~m} / \mathrm{s}$.

Представленная в настоящей работе методика использовалась для построения проекций изображений взрыва метеора и распространяющегося следа на поверхность Земли по данным двух вышеописанных камер. Использование двух камер позволяет построить трехмерное изображение объекта. Для этого был создан набор проекций в некотором диапазоне высот и проведено сопоставление проекций, построенных по данным различных камер. Если географические координаты части метеора или ДМС в данном наборе совпадают на определенной высоте, это означает, что данная часть объекта сосредоточена на этой высоте. Стереоскопическим методом анализа проекций было определено, что метеор зажигается на высоте около $111 \mathrm{~km}$ и гаснет на высоте $78 \mathrm{~km}$, а полная длина трека составляет около $38 \mathrm{~km}$ (рис. 5). При этом ошибка определения высот лежит в пределах $\pm 1.2 \mathrm{~km}$.

Аналогичный анализ проекции метеорного следа показал, что он сосредоточен в диапазоне $\sim 88-97 \mathrm{~km}$ и распространялся преимущественно в южном направлении вверх вплоть до $\sim 95-106 \mathrm{~km}$. Распространение следа в восточном направлении происходило на высоте $\sim 88 \mathrm{~km}$ со средней скоростью около $30 \mathrm{~m} / \mathrm{s}$. В южном направлении след распространялся преимущественно на высоте около $94 \mathrm{~km}$ со средней скоростью около $84 \mathrm{~m} / \mathrm{s}$. В западном направлении средняя скорость движения следа была около $52 \mathrm{~m} / \mathrm{s}$. Следует отметить, что скорости, полученные по данным оптических наблюдений описанной выше методикой, оказались близки к значениям скоростей ионизационного следа, полученных в работе [28] по данным ионозонда.

\section{Заключение}

Представленная методика позволяет достаточно точно идентифицировать звезды на кадрах широкоугольной системы, получать пространственные параметры зарегистрированных событий и соответственно строить проекции на поверхность Земли. Погрешности при определении идентифицированных звезд не превышают $0.8^{\circ}$. Использование двух или нескольких камер, разнесенных на некоторое расстояние, позволяет создавать стереоскопическое представление различных событий, позволяющее более полно оценивать наблюдаемое событие. После обработки изображений ДМС с помощью представленного алгоритма были получены следующие пространственные характеристики следа: высоты распространения - от 88 до $106 \mathrm{~km}( \pm 1.2 \mathrm{~km})$, направление распространения преимущественно южное. Также рассчитаны скорости следа в основных направлениях: южном, западном и восточном - 84, $\sim 52$ и $\sim 30 \mathrm{~m} / \mathrm{s}$ соответственно.
Данную методику в дальнейшем предполагается использовать для оценки трехмерных координат объектов, наблюдаемых с помощью широкоугольных оптических систем, таких как метеоры, проявления акустикогравитационных волн и т.п.

\section{Финансирование работы}

Работа выполнена при финансовой поддержке Минобрнауки России (субсидия № 075-ГЗ/Ц3569/278) и РФФИ в рамках научного проекта № 19-35-90093.

\section{Конфликт интересов}

Авторы заявляют, что у них нет конфликта интересов.

\section{Список литературы}

[1] I.S. Izmailov, M.L. Khovricheva, M.Yu. Khovrichev, O.V. Kiyaeva, E.V. Khrutskaya, L.G. Romanenko, E.A. Roshchina, K. Maslennikov, O.A. Kalinichenko. Astron. Lett., 36 (5), 349 (2010).

[2] D. Lang, D.W. Hogg, K. Mierle, M. Blanton, S. Roweis. Astron. J., 139 (5), 1782 (2010). DOI: $10.1088 / 0004-6256 / 139 / 5 / 1782$.

[3] T. Aso, T. Hashimoto, M. Abe, T. Ono, M. Ejiri. J. Geomagn. Geoelectr., 42 (5), 579 (1990). https://doi.org/10.5636/jgg.42.579

[4] D. Megan Gillies, D. Knudsen, E. Donovan, B. Jackel, R. Gillies, E. Spanswick. J. Geophys. Res. Space Phys., 122, 8181 (2017). DOI: 10.1002/2016JA023758

[5] B.J. Jackel, F. Creutzberg, E.F. Donovan, L.L. Cogger. Sodankylä Geophys. Observ. Publ., 92, 97 (2003).

[6] C. Störmer. Comptes Rendus de l'Académie des Sciences, 150, 1631 (1910).

[7] G. Witt. Tellus, 14 (1), 1 (1962). DOI: $10.3402 /$ tellusa.v14i1.9524

[8] G. Romick, A. Belon. Planet. Space Sci., 15 (11), 1695 (1967). https://doi.org/10.1016/0032-0633(67)90008-6

[9] H.C. Stenbaek-Nielsen, T.J. Hallinan. J. Geophys. Res., 84, 3257 (1979). https://doi.org/10.1029/JA084iA07p03257

[10] M.N. Kouahla, G. Moreels, M. Faivre, J. Clairemidi, J.W. Meriwether, G.A. Lehmacher, E. Vidal, O. Veliz. Adv. Space Res., 45, 260 (2010). https://doi.org/10.1016/j.asr.2009.10.015

[11] T. Pedersen, B. Gustavsson, E. Mishin, E. MacKenzie, H.C. Carlson, M. Starks, T. Mills. Geophys. Res. Lett., 36 (18), (2009). DOI: 10.1029/2009GL040047

[12] A.V. Shindin, V.V. Klimenko, D.A. Kogogin, A.B. Beletsky, S.M. Grach, I.A. Nasyrov, E.N. Sergeev. Radiophys. Quantum El., 60 (11), 849 (2018). DOI: $10.1007 / \mathrm{s} 11141-018-9852-0$

[13] R. Danescu, F. Oniga, V. Turcu, O. Cristea. Sensors, 12, 12940 (2012). DOI:10.3390/s121012940

[14] S. Shah, J.K. Aggarwal. Pattern Recogn., 29 (11), 1775 (1996). https://doi.org/10.1016/0031-3203(96)00038-6

[15] D. Barghini, D. Gardiol, A. Carbognani, S. Mancuso. Astron. Astrophys., 626, A105 (2019).

https://doi.org/10.1051/0004-6361/201935580 
[16] J. Wang, F. Shi, J. Zhang, Y. Liu. Pattern Recogn., 41 (2), 607 (2008). https://doi.org/10.1016/j.patcog.2007.06.012

[17] F. Huang, Y. Wang, X. Shen, C. Lin, Y. Chen. Appl. Opt., 51 (34), 8169 (2012). https://doi.org/10.1364/AO.51.008169

[18] S.S. Beauchemin, R. Bajcsy. In: Multi-Image Analysis. Lecture Notes in Computer Science, ed. by R. Klette, G. Gimel'farb, T. Huang (Springer, Berlin, Heidelberg, 2001). https://doi.org/10.1007/3-540-45134-X_1

[19] Multidimensional Gaussian filter [Электронный ресурс]KD Режим доступа: https://docs.scipy.org/doc/scipy/reference/ generated/scipy.ndimage.gaussian_filter.html

[20] C. Ceplecha. Bull. Astron. Inst. Czech., 38, 222 (1987).

[21] J. Borovička, P. Spurný, J. Keclíková. Astron. Astrophys.Suppl. Ser., 112, 173 (1995).

[22] S. Bannister, L. Boucheron, D. Voelz. Publ. Astron. Soc. Pac., 125, 1108 (2013).

[23] J.J. Moré. In: Numerical Analysis. Lecture Notes in Mathematics, ed. by G.A. Watson (Springer, Berlin, Heidelberg, 1978), v. 630 .

[24] ephem 4.0.0.2 [Электронный ресурс] Режим доступа: https://pypi.org/project/ephem/

[25] В.П. Морозов. Курс сфероидической геодезии (Недра, М., 1979)

[26] Keo Sentinel Wide-Angle Monochromatic Imager [Электронный ресурс] Режим доступа: http://atmos.iszf.irk.ru/ru/data/keo

[27] А.В. Михалев, А.Б. Белецкий, Р.В. Васильев, М.В. Еселевич, К.И. Иванов, Е.С. Комарова, А.В. Подлесный, С.В. Подлесный, Т.Е. Сыренова. В сб.: Сборник тезисов докладов XVI Всероссийской открытой конференции „Современные проблемы дистанщионного зондирования Земли из космоса (М., ИКИ РАН, 2018), с. 476. DOI 10.21046/2070-16DZZconf-2018a

[28] А.В. Михалев, А.Б. Белецкий, Р.В. Васильев, М.В. Еселевич, К.И. Иванов, Е.С. Комарова, А.В. Подлесный, С.В. Подлесный, Т.Е. Сыренова. Солнечно-земная физика, 5 (3), 130 (2019). DOI: 10.12737/szf-53201913

[29] К.И. Иванов, Е.С. Комарова, Р.В. Васильев, М.В. Еселевич, А.В. Михалев. Солнечно-земная физика, 5 (1), 100 (2019). DOI: $10.12737 /$ szf-51201911 